\title{
O MODELO IFLA LIBRARY REFERENCE MODEL E O LINKED DATA
}

\section{IFLA LIBRARY REFERENCE MODEL AND LINKED DATA}

Ana Carolina Simionato Arakakia

\begin{abstract}
RESUMO
Introdução: Diante da nova proposta do modelo IFLA LRM, os modelos conceituais da família FR se consolidam a fim de facilitar a criação de relacionamentos entre as entidades e na definição de atributos. Ademais, o LRM traz mudanças em sua aproximação com a disponibilização de dados e com a Web Semântica. Objetivo: Dessa forma, objetiva-se apresentar o modelo IFLA LRM e relacioná-lo com as novas tecnologias disponíveis para possibilitar o instanciamento do modelo a partir dos conceitos da Web Semântica. Metodologia: Caracteriza-se por uma pesquisa qualitativa e exploratória, por meio de um levantamento bibliográfico sobre o modelo IFLA LRM e os princípios Linked Data, com o recorte da pesquisa em trabalhos internacionais e nacionais desde o ano de publicação do modelo LRM. Resultados: A principal contribuição é explanada pela instanciação da classe 'obra' e são apresentadas discussões sobre as relações do modelo LRM. Conclusões: Considera-se que, pela instanciação dos atributos do domínio 'Irmer', os estudos poderão ser significativos para a aplicação em catálogos digitais e que tenham o intuito da abertura dos dados bibliográficos.
\end{abstract}

Descritores: IFLA Library Reference Model. Linked Data. Web Semântica. Modelos conceituais.

\section{INTRODUÇÃO}

Passados mais de dez anos da publicação dos Requisitos Funcionais para Registros Bibliográficos (FRBR), o eixo de representação e organização da informação se deparou com diversas transformações pelo viés dos modelos conceituais.

O repensar dos processos de Catalogação tornou-se fundamental para

\footnotetext{
a Doutora em Ciência da Informação pela Universidade Estadual Paulista (UNESP). Professora do Programa de Pós-Graduação em Ciência da Informação da Universidade Federal de São Carlos (PPGCI/UFSCar). E-mail: acsimionato@ufscar.br.
} 
refletir sobre os atuais instrumentos de representação. A considerar nas últimas décadas um período de desenvolvimento tecnológico para tratamento de recursos informacionais, juntamente com a necessidade de adequar os catálogos às necessidades dos usuários, surgiram novos padrões e diretrizes para a Catalogação.

Neste contexto, a Catalogação deve ser vista além da técnica, como uma disciplina que reflita a forma teórica e prática dos processos de representação e organização da informação. Isto pois, de acordo com ALCTS (2017), o catalogador requer conhecimentos sobre: os princípios básicos de catalogação e metadados; de sistemas e tecnologias; das tendências na profissão de catalogação e metadados; habilidades em aplicar estruturas conceituais, padrões e princípios dentro de um sistema bibliográfico; aplicar padrões universais em um contexto local; conhecer a integração, o mapeamento e a transformação de metadados em um sistema bibliográfico.

Santos (2010) complementa que nos ambientes multidisciplinares "[...] o catalogador tem a vantagem intelectual de ser o conhecedor da essência dos processos de análise e tratamento do conhecimento registrado.". Entretanto, a autora explica ser fundamental o catalogador apropriar-se das ferramentas tecnológicas disponíveis para agilizar os processos decisivos, e para o estabelecimento da organização e do tratamento da informação (SANTOS, 2010, p. 189).

Com isso, criou-se uma nova perspectiva para descrição de objetos nas bibliotecas. Isto em razão da necessidade de repensar a descrição, ainda no processo de estruturação dos sistemas de informação, ou seja, no planejamento e na modelagem dos bancos de dados. Para Fusco (2011, p. 68), a modelagem conceitual

[...] fornece uma visão mais próxima do modo como os usuários visualizam os dados e definem especificações necessárias à qualidade das informações do ambiente informatizado a ser criado. Por desenvolver uma atividade de abstração de nível superior e não depender de tecnologia, o profissional da informação deve se preocupar como os elementos de dados definidos no escopo do domínio estarão conceitualmente representados e relacionados e, para tanto, deve conhecer uma metodologia de construção de modelos conceituais.

Diante desse contexto, em 1998 publicaram o Functional Requirements 
for Bibliographic Record (FRBR), cujo foco era a construção de um modelo para registros bibliográficos. Posteriormente, foram divulgados o Functional Requirements for Authority Data (FRAD), em 2009, com base na organização dos registros de autoridade. Em 2010, foi publicado o Functional Requirements for Subject Authority Data (FRSAD), a tratar especificamente dos assuntos. Contudo, observou-se inconsistências para implementação e necessidades de harmonização desses modelos. Dessa forma, surgiu o IFLA Library Reference Model - IFLA LRM.

A partir disso, o objetivo deste artigo é apresentar o modelo IFLA LRM e relacioná-lo com as novas tecnologias disponíveis para possibilitar o instanciamento do modelo, a partir dos conceitos da Web Semântica.

Derivado de um estudo inicial (SIMIONATO, 2017), este artigo é caracterizado por uma pesquisa exploratória da literatura disponível sobre o tema proposto, a permitir a construção de um conhecimento teórico sobre a Catalogação, frente às tecnologias da Web Semântica. Consultaram-se fontes primárias, secundárias e terciárias nas bases de dados da Ciência da Informação, tais como Base de Dados Referenciais de Artigos de Periódicos em Ciência da Informação (BRAPCl), e periódicos da área de Ciência da Informação, no Portal de Periódicos da CAPES. Além disso, foram examinadas as bases internacionais Web of Science e Scientific Electronic Library Online (SciELO), bem como o Google Scholar. Sendo os termos de busca aplicados: "IFLA LRM", "LRM", "Library Reference Model', "Linked Data", "Web Semantic", "Web Semântica", "Web Semántica", "dados ligados", "dados conectados", "dados interligados", "Linked Open Data", "datos conectados" e "datos vinculados". O recorte da pesquisa abrange outras publicadas internacionalmente e no Brasil, nos idiomas em português, em espanhol e em inglês.

O texto abrange, além da seção de introdução, outra a qual expõe o modelo IFLA LRM. Por conseguinte, argumenta-se sobre os princípios e as tecnologias que envolvem o Linked Data. Por fim, discute-se estruturação do modelo IFLA LRM, a partir das tecnologias do Linked Data, e considerações finais. 


\section{IFLA LIBRARY REFERENCE MODEL}

O modelo IFLA Library Reference Model: a conceptual model for bibliographic information foi o resultado de uma harmonização e da ampliação da família de requisitos funcionais (Functional Requirements - FR) da International Federation of Library Associations and Institutions (IFLA). A família FR, iniciada em 1998, é composta pelos modelos conceituais: Functional Requirements for Bibliographic Record (FRBR) para registros bibliográficos; Functional Requirements for Authority Data (FRAD) para registros de autoridade; e o Functional Requirements for Subject Authority Data (FRSAD) para registros de assunto.

Os três modelos cresceram com escopos distintos e com desenvolvedores diferentes, embora compartilhem de algumas soluções semelhantes. Mesmo com diferenças, os direcionamentos para a criação do LRM foram realizados de forma consistente e padronizada, a fim de uma consolidação dos compromissos ontológicos, já delineados no primeiro FR (RIVA; BCEUF; ŽUMER, 2017a).

Conforme Riva, Bœuf e Žumer (2017a, p. 09) afirmam na divulgação do modelo LRM, em 2017, é uma publicação para a definição de um modelo de referência conceitual para fornecer uma estrutura para a análise de metadados não administrativos relacionados aos recursos das bibliotecas. É considerado como "[...] um modelo conceitual de alto nível e, como tal, pretende ser um guia ou base para formular regras de catalogação e implementar sistemas bibliográficos.". No texto, os autores reforçam a ideia para qualquer reprodução do modelo. Deve-se ser respeitado pelas estruturas básicas das entidades e ser fiel aos relacionamentos entre as entidades (incluindo as limitações das cardinalidades), bem como o estabelecimento das conexões entre os atributos.

Outro ponto de destaque para o modelo LRM é que

[...] um critério importante para a manutenção ou criação de uma entidade foi que ela precisava se mostrar necessária como domínio ou abrangência de pelo menos um relacionamento significativo ou possuir pelo menos um atributo relevante que não pudesse ser generalizado logicamente para uma superclasse da entidade. Um fator importante na avaliação de relacionamentos e atributos foi determinar se eles poderiam ser 
generalizados, incluindo se eles poderiam ser declarados em um nível superior utilizando uma entidade de superclasse. As entidades foram adicionadas desde que elas pudessem ser utilizadas para otimizar o modelo, permitindo a redução de relacionamentos ou atributos. (RIVA; BOEUF; ŽUMER, 2017a, p. 11).

Por esses apontamentos iniciais, ressalta-se que o IFLA LRM possui o escopo funcional por meio das tarefas do usuário, definidas do ponto de vista e das necessidades do usuário final, e não nas operações da biblioteca. As tarefas do usuário do modelo IFLA LRM podem ser visualizadas no Quadro 1.

\section{Quadro 1 - Tarefas do usuário do modelo IFLA LRM}

\begin{tabular}{|c|l|}
\hline Encontrar & $\begin{array}{l}\text { Reunir informações sobre um ou mais recursos de interesse pesquisando sobre } \\
\text { qualquer critério relevante; }\end{array}$ \\
\hline Identificar & $\begin{array}{l}\text { Compreender claramente a natureza dos recursos encontrados e distinguir entre } \\
\text { recursos semelhantes; }\end{array}$ \\
\hline Selecionar & $\begin{array}{l}\text { Determinar a adequação dos recursos encontrados e aceitar ou rejeitar recursos } \\
\text { específicos; }\end{array}$ \\
\hline Obter & Acessar o conteúdo do recurso; \\
\hline Explorar & $\begin{array}{l}\text { Descobrir recursos utilizando os relacionamentos entre eles e, assim, } \\
\text { contextualizá-los. }\end{array}$ \\
\hline
\end{tabular}

Fonte: Riva, Bœuf e Žumer (2017a, p. 16).

Além da identificação das cinco tarefas do usuário, o documento direciona para outras orientações. Uma delas é sobre a ordem de execução das tarefas. Segundo os autores, a busca pelos recursos informacionais não segue a listagem conforme a apresentação do Quadro 1. Isto é, o processo de busca, aquisição e compreensão do recurso é iterativo e pode ser tangencial a qualquer momento, ou seja, o usuário pode identificar e selecionar ao mesmo tempo. Na publicação do LRM, os autores Riva, Bœuf e Žumer (2017a) ponderam que no contexto dos requisitos funcionais, a tarefa de explorar a navegação deve ser enfatizada para um sistema bibliográfico moderno, o qual suporte a navegação e, consequentemente, a uma descoberta imprevista pelo usuário.

Com relação à estrutura, o modelo IFLA LRM adotou uma abordagem geral e metodológica do estudo original sobre FRBR. Riva, Bœuf e Žumer (2017a) relatam do detalhamento do estudo neste processo de harmonização da família FR. Realizou-se uma análise detalhada de todas as entidades, como 
visualizado no Quadro 2, uma vez que incluem entidades semelhantes entre os modelos. Por exemplo: obra, expressão, manifestação e item, entidades similares, como 'Nomen' no FRSAD e 'Nome' no FRAD, ou ainda completamente diferentes, como pessoa no FRBR e pessoa no FRAD (ŽUMER, 2019).

Com maiores detalhes, o Quadro 2 faz uma comparação entre as entidades dos modelos conceituais da família FR, baseado no mapeamento de Riva, Bœuf e Žumer (2017b, p. 09-13). São expostas as entidades e a harmonização realizada na definição das entidades. O conteúdo do quadro que está destacado por um * (asterisco) refere-se a resultados do mapeamento como subclasses. Nas três primeiras colunas há as entidades da família FR e na última as entidades do modelo IFLA LRM.

Quadro 2 - Comparação das entidades dos modelos conceituais

\begin{tabular}{|c|c|c|c|}
\hline FRBR & FRAD & FRSAD & IFLA LRM \\
\hline $\begin{array}{l}\text { Obra: uma distinta } \\
\text { criação intelectual ou } \\
\text { artística. }\end{array}$ & $\begin{array}{l}\text { Obra: uma distinta } \\
\text { criação intelectual ou } \\
\text { artística. (como no } \\
\text { FRBR) }\end{array}$ & $\begin{array}{l}\text { Obra: uma distinta } \\
\text { criação intelectual ou } \\
\text { artística. (como no } \\
\text { FRBR) }\end{array}$ & $\begin{array}{l}\text { Obra: uma distinta } \\
\text { criação intelectual ou } \\
\text { artística. }\end{array}$ \\
\hline $\begin{array}{l}\text { Expressão: realização } \\
\text { intelectual ou artística } \\
\text { de uma 'Obra' na forma } \\
\text { alfanumérica, musical } \\
\text { ou notação } \\
\text { coreográfica, som, } \\
\text { imagem, objeto, } \\
\text { movimento, etc., ou } \\
\text { outra forma de } \\
\text { combinação. }\end{array}$ & $\begin{array}{l}\text { Expressão: realização } \\
\text { intelectual ou artística } \\
\text { de uma 'Obra' na forma } \\
\text { alfanumérica, musical } \\
\text { ou notação } \\
\text { coreográfica, som, } \\
\text { imagem, objeto, } \\
\text { movimento, etc., ou } \\
\text { outra forma de } \\
\text { combinação. (como no } \\
\text { FRBR) }\end{array}$ & - & $\begin{array}{c}\text { Expressão: uma } \\
\text { combinação específica } \\
\text { de sinais que } \\
\text { transmitem conteúdo } \\
\text { intelectual ou artístico }\end{array}$ \\
\hline $\begin{array}{l}\text { Manifestação: a } \\
\text { personificação física da } \\
\text { Expressão e da Obra. }\end{array}$ & $\begin{array}{l}\text { Manifestação: a } \\
\text { personificação física da } \\
\text { Expressão e da Obra. } \\
\text { (como no FRBR) }\end{array}$ & - & $\begin{array}{c}\text { Manifestação: Um } \\
\text { conjunto de todos os } \\
\text { suportes que } \\
\text { supostamente } \\
\text { compartilham as } \\
\text { mesmas características } \\
\text { do conteúdo intelectual } \\
\text { ou artístico e aspectos } \\
\text { da forma física. Esse } \\
\text { conjunto é definido pelo } \\
\text { conteúdo geral e pelo } \\
\text { plano de produção para } \\
\text { seu suporte ou } \\
\text { suportes. }\end{array}$ \\
\hline $\begin{array}{l}\text { Item: um único } \\
\text { exemplar de uma } \\
\text { 'Manifestação'. }\end{array}$ & $\begin{array}{c}\text { Item: um único } \\
\text { exemplar de uma } \\
\text { 'Manifestação'. (como } \\
\text { no FRBR) }\end{array}$ & - & $\begin{array}{l}\text { Item: Um ou vários } \\
\text { objetos que carregam } \\
\text { sinais destinados a } \\
\text { transmitir conteúdo }\end{array}$ \\
\hline
\end{tabular}




\begin{tabular}{|c|c|c|c|}
\hline & & & intelectual ou artístico. \\
\hline- & - & - & $\begin{array}{l}\text { Agente: uma entidade } \\
\text { capaz de ações } \\
\text { deliberadas, de cessão } \\
\text { de direitos e de ser } \\
\text { responsabilizada por } \\
\text { suas ações. }\end{array}$ \\
\hline Pessoa: um indivíduo. & $\begin{array}{l}\text { Pessoa: um indivíduo } \\
\text { ou uma pessoa ou uma } \\
\text { identidade estabelecida } \\
\text { ou adotada por um } \\
\text { indivíduo ou grupo. }\end{array}$ & - & $\begin{array}{l}\text { Pessoa: um ser } \\
\text { humano individual. }\end{array}$ \\
\hline- & - & - & $\begin{array}{l}\text { Agente coletivo: uma } \\
\text { reunião ou organização } \\
\text { de pessoas com um } \\
\text { nome específico e } \\
\text { capaz de atuar como } \\
\text { uma unidade. }\end{array}$ \\
\hline- & $\begin{array}{c}\text { Família: duas ou mais } \\
\text { pessoas relacionadas } \\
\text { por nascimento, } \\
\text { casamento, adoção, } \\
\text { união civil ou situação } \\
\text { legal semelhante, ou } \\
\text { que de outra forma se } \\
\text { apresentam como uma } \\
\text { família. }\end{array}$ & - & $\begin{array}{c}\text { * Família: poderá ser } \\
\text { utilizada como uma } \\
\text { subclasse de 'Agente } \\
\text { coletivo' }\end{array}$ \\
\hline $\begin{array}{l}\text { Entidade Coletiva: uma } \\
\text { organização ou grupo } \\
\text { de indivíduos e/ou } \\
\text { organizações agindo } \\
\text { como uma unidade. }\end{array}$ & $\begin{array}{l}\text { Entidade Coletiva: uma } \\
\text { organização ou grupo } \\
\text { de pessoas e/ou } \\
\text { organizações } \\
\text { identificadas por um } \\
\text { nome particular agindo } \\
\text { como uma unidade. }\end{array}$ & - & $\begin{array}{l}\text { * Entidade coletiva: } \\
\text { poderá ser utilizado } \\
\text { como uma subclasse } \\
\text { de 'Agente coletivo' }\end{array}$ \\
\hline- & - & $\begin{array}{l}\text { Thema: qualquer } \\
\text { entidade usada como } \\
\text { um assunto de um } \\
\text { trabalho. }\end{array}$ & $\begin{array}{l}\text { Res: qualquer entidade } \\
\text { no universo do } \\
\text { discurso. }\end{array}$ \\
\hline $\begin{array}{l}\text { Conceito: uma noção } \\
\text { ou ideia abstrata. }\end{array}$ & $\begin{array}{c}\text { Conceito: uma noção } \\
\text { ou ideia abstrata (como } \\
\text { no FRBR) }\end{array}$ & - & - \\
\hline $\begin{array}{c}\text { Objeto: uma coisa } \\
\text { material. }\end{array}$ & $\begin{array}{l}\text { Objeto: uma coisa } \\
\text { material (como no } \\
\text { FRBR) }\end{array}$ & - & - \\
\hline $\begin{array}{l}\text { Evento: uma ação ou } \\
\text { ocorrência. }\end{array}$ & $\begin{array}{c}\text { Evento: uma ação ou } \\
\text { ocorrência (como no } \\
\text { FRBR) }\end{array}$ & - & - \\
\hline Lugar: uma localização. & $\begin{array}{l}\text { Lugar: uma localização } \\
\text { (como no FRBR) }\end{array}$ & - & $\begin{array}{l}\text { Lugar: uma extensão } \\
\text { de espaço } \\
\text { determinada. }\end{array}$ \\
\hline- & - & - & Intervalo de tempo: \\
\hline
\end{tabular}

Inf. Inf., Londrina, v. 25, n. 3, p. 163 - 186, jul./set. 2020. 


\begin{tabular}{|c|c|c|c|}
\hline & & & $\begin{array}{l}\text { uma extensão temporal } \\
\text { que possui um início, } \\
\text { um fim e uma duração. }\end{array}$ \\
\hline - & $\begin{array}{c}\text { Nome: um } \\
\text { personagem, palavra } \\
\text { ou grupo de palavras } \\
\text { e/ou caracteres pelos } \\
\text { quais uma entidade é } \\
\text { conhecida. }\end{array}$ & $\begin{array}{l}\text { Nomem: qualquer sinal } \\
\text { ou sequência de sinais } \\
\text { (caracteres } \\
\text { alfanuméricos, } \\
\text { símbolos, som etc.) que } \\
\text { um Thema é conhecido } \\
\text { por, referido para, ou } \\
\text { endereçado como. }\end{array}$ & $\begin{array}{l}\text { Nomem: uma } \\
\text { associação entre uma } \\
\text { entidade e uma } \\
\text { designação. }\end{array}$ \\
\hline- & $\begin{array}{l}\text { Identificador: um } \\
\text { número, código, } \\
\text { palavra, frase, logotipo, } \\
\text { dispositivo etc., ou seja, } \\
\text { associado a uma } \\
\text { entidade. Serve para } \\
\text { diferenciar essa } \\
\text { entidade de outras, } \\
\text { dentro do domínio em } \\
\text { que o identificador é } \\
\text { atribuído. }\end{array}$ & - & $\begin{array}{l}\text { * Identificador: poderá } \\
\text { ser utilizado como uma } \\
\text { subclasse de 'Nomen' }\end{array}$ \\
\hline- & $\begin{array}{l}\text { Ponto de acesso } \\
\text { controlado: um nome, } \\
\text { termo, código, entre } \\
\text { outros, sob o qual um } \\
\text { registro bibliográfico ou } \\
\text { de autoridade será } \\
\text { encontrado. }\end{array}$ & - & $\begin{array}{c}\text { *Ponto de acesso } \\
\text { controlado: poderá ser } \\
\text { utilizada como uma } \\
\text { subclasse de 'Agente } \\
\text { coletivo' }\end{array}$ \\
\hline- & $\begin{array}{l}\text { Regras: um conjunto de } \\
\text { instruções relativas à } \\
\text { formulação e/ou } \\
\text { registro dos pontos de } \\
\text { acesso controlados. }\end{array}$ & - & - \\
\hline- & $\begin{array}{l}\text { Agência: uma } \\
\text { organização } \\
\text { responsável por criar e } \\
\text { modificar um ponto de } \\
\text { acesso controlado. }\end{array}$ & - & - \\
\hline
\end{tabular}

Fonte: Elaborado pela autora.

O Quadro 2 faz uma comparação entre as entidades da família FR. Algumas delas, no modelo IFLA LRM, foram herdadas ou derivadas, outras modificadas, enquanto outras ficaram obsoletas. As entidades no modelo IFLA LRM são consideradas como categorias abstratas (classes) de objetos conceituais conectados por relacionamentos, sendo que suas características são descritas pelos atributos.

Nesse viés, é importante relembrar que o propósito do FRBR é auxiliar o usuário enquanto este realiza atividades ao consultar os registros bibliográficos 
presentes nos catálogos. Portanto,

O estudo não faz suposições a priori sobre o registro bibliográfico em si mesmo, nem em termos de conteúdo, ou de estrutura. Ele emprega uma abordagem focada no usuário para analisar os requisitos de dados, na medida em que se esforça para definir, de maneira sistemática, aquilo que o usuário espera encontrar informações a respeito, num registro bibliográfico, e como aquela informação é usada. (IFLA STUDY GROUP ON THE FUNCTIONAL REQUIREMENTS, 2009, p. 03, tradução nossa).

A principal e maior contribuição da família FR é a reformulação intrínseca dos registros, conforme a estruturação de cada modelo, o que evita a redundância de dados. Neste momento, o modelo LRM vem justamente harmonizar essa estruturação e, ainda, criar uma base única para a modelagem conceitual de registros bibliográficos. Inclui os dados de autoridade, assunto e os bibliográficos, em um sentido amplo e geral.

Esse eixo entre entidades, relacionamentos e atributos do modelo LRM decorre da modelagem conceitual de Entidade-Relacionamento $(E-R)$, originária da Ciência da Computação para o desenvolvimento de banco de dados. Presente em todos os modelos da família FR, essa modelagem é representada por notações ou diagramas que expressam os relacionamentos do modelo.

Todavia, destaca-se que o primeiro modelo, o FRBR, utilizou a diagramação da modelagem $\mathrm{E}-\mathrm{R}$, mas não aplicou a técnica original em todos os seus aspectos (MAXWELL, 2008). Nesse sentido, Karen Coyle faz a seguinte análise:

Os diagramas de E-R do FRBR apresentam um modelo de nível macro que inclui somente o que são considerados como sendo relacionamentos entre entidades. Outros relacionamentos entre entidades bibliográficas são definidos no texto, tais como relacionamentos obra/obra. Estes não aparecem nos diagramas do relatório final dos FRBR, portanto, é apresentando um quadro incompleto do modelo bibliográfico real descrito no texto. (COYLE, 2016, p. 78, tradução nossa).

A diagramação é essencial para a viabilidade computacional do modelo e, ainda, para a implementação em sistemas e bases de dados. Esse ponto é destacado nesse momento, em razão do modelo LRM descrever a superclasse da entidade 'Res' com todas as outras e com o relacionamento IsA ("is a"), derivada de uma hierarquia que pode adicionar atributos descritivos específicos 
para as entidades da subclasse.

A superclasse da entidade 'Res' pode ser mais bem explicitada na visualização do Quadro 3, com uma hierarquia de classe, ou is $A$ para entidades.

Quadro 3 - Hierarquia das entidades no IFLA LRM

\begin{tabular}{|c|c|c|}
\hline Nível Superior & Segundo Nível & Terceiro nível \\
\hline LRM-E1 Res & -- & -- \\
\hline-- & LRM-E2 Obra & -- \\
\hline-- & LRM-E3 Expressão & -- \\
\hline-- & LRM-E4 Manifestação & -- \\
\hline-- & LRM-E5 Item & LRM-E7 Pessoa \\
\hline-- & LRM-E6 Agente & -- \\
\hline-- & -- & -- \\
\hline-- & -- & LRM-E8 Agente Coletivo \\
\hline-- & LRM-E9 Nomen \\
\hline-- & LRM-E10 Lugar & - \\
\hline-- & LRM-E11 Intervalo de & \\
\hline & Tempo & \\
\hline
\end{tabular}

Fonte: Riva, Bœuf e Žumer (2017a, p. 18).

Outro destaque para o modelo LRM é a possibilidade de identificar as entidades que pertencem a algum relacionamento no modelo. Como é descrito na publicação:

As entidades servem como domínios e abrangências dos relacionamentos destacados no modelo. Os atributos definidos para cada entidade servem para definir melhor suas características. Uma entidade é uma classe abstrata de objetos conceituais; existem muitas instâncias de cada entidade que são descritas em dados bibliográficos, de coleções ou de autoridade. Uma entidade pode ser declarada uma superclasse de outras entidades que, por sua vez, possuem uma relação de subclasse com essa entidade. Qualquer instância de uma entidade de subclasse é também uma instância da superclasse. Isto forma parte da estrutura do modelo entidade-relacionamento expandido e pode ser expresso como "is a" (ou isA). (RIVA; BOEUF; ŽUMER, 2017a, p. 19).

Destarte, essa é uma das novidades estruturais do LRM. No qual, a hierarquia de classes e subclasses, atributos e relacionamentos são declarados em um nível mais alto, e são herdados por todas as subclasses, e não precisam ser repetidos em níveis mais baixos (ŽUMER, 2019), como apresentado na Figura 1. 
Figura 1 - Os relacionamentos e as entidades do modelo IFLA LRM

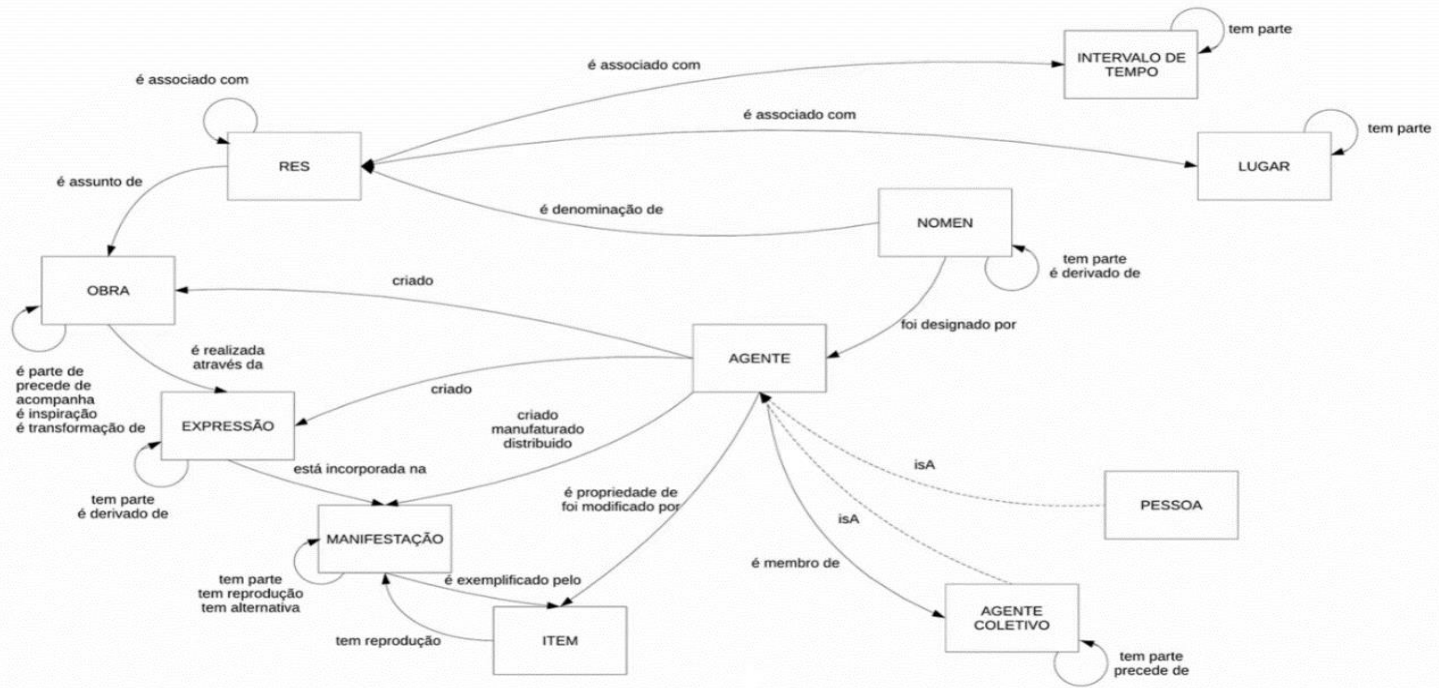

Fonte: Riva, Bœuf e Žumer (2017a, p. 88).

As entidades 'Obra', 'Expressão', 'Manifestação' e 'Item', do modelo IFLA LRM, permanecem as mesmas do Grupo 1 do FRBR. No entanto, há algumas diferenças nas definições e notas de escopo, como visualizadas anteriormente no Quadro 2. No modelo IFLA LRM, a entidade 'Agente' é caracterizada como uma superclasse. Do mesmo modo, 'Agente' também é uma entidade do Grupo 2 do FRBR e do FRAD, e a entidade 'Pessoa' do FRAD. Como superclasse, a entidade 'Agente' incorpora as entidades 'Pessoa' e 'Agente coletivo'. Destacase que no LRM pessoas fictícias, literárias e lendárias não estão incluídas. Nesses casos, eles poderão ser temas da entidade 'Obra'. No LRM mantiveramse as entidades 'Thema' e 'Nomen' do modelo FRSAD, porém com outras denominações. 'Thema' foi renomeado para 'Res', sendo esta uma superclasse de todas as entidades do modelo IFLA LRM e evita a restrição implícita à relação do sujeito. Enquanto a entidade 'Nomen' é atribuída para se referir a uma instância da superclasse 'Res'. Por fim, as últimas entidades criadas foram para os aspectos temporais e espaciais no modelo LRM, que são 'Local' e 'Intervalo de tempo'.

Os atributos são caracterizados como instâncias específicas de uma entidade e podem ser definidos pelo modelo LRM. Podem não ser necessários para qualquer instância, mas são capazes de serem registrados quando os dados são considerados relevantes para o propósito da aplicação do LRM (RIVA; 
BCEUF; ŽUMER, 2017a).

Os dados de atributos podem ser registrados de acordo com uma lista ou vocabulário controlado, uma linguagem natural ou um script da preferência da agência ou organização que registra os dados. "Os atributos apresentados em cada entidade são representativos e não devem ser considerados de forma alguma uma lista exaustiva de atributos que podem ser considerados úteis em uma aplicação específica." (RIVA; BCEUF; ŽUMER, 2017a, p. 38).

Riva, Bœuf e Žumer (2017) detalham que em uma aplicação do LRM é possível definir atributos adicionais para registrar dados relevantes ou com um nível maior ou menor de granularidade. Dessa forma, os atributos podem ser multivalorados e os atributos de categoria servem para subdividir ou subcategorizar entidades, conforme a relevância para o esquema em uma aplicação específica. Assim, vários tipos de categorizações independentes são possíveis de serem aplicados a uma entidade em uma determinada implementação.

Com essa consolidação do IFLA LRM, é visto no relatório o ensejo para o emprego dos princípios Linked Data nos registros bibliográficos. Assim, cria-se possibilidades de aplicações derivadas destes dados e reduz a ambiguidade gerada dos modelos e padrões tradicionais.

\section{PRINCÍPIOS LINKED DATA}

Os princípios Linked Data são orientações para boas práticas de publicação de dados na Web Semântica. Eles possibilitam a otimização de processos como uso, reuso, compartilhamento, acesso, descoberta, proveniência e interoperabilidade dos dados. A concepção do Linked Data foi apresentada por Berners-Lee, em 2006, em quatro princípios que servem como sugestões para estruturação de dados. São eles:

1 Use URIs como nomes para as coisas;

2 Use HTTP URIs para que as pessoas possam procurar esses nomes;

3 Quando alguém procurar um URI, fornecer informações úteis, usando os padrões (RDF, SPARQL);

4 Incluir links para outros URIs, para que eles possam descobrir mais coisas. 
Berners-Lee (2006) utilizou um sistema que classifica em cinco estrelas a disponibilização de Linked Open Data. Arakaki (2016) esclarece que o Linked Data está relacionado à interoperabilidade técnica dos dados, enquanto o Linked Open Data refere-se à interoperabilidade legal, ou seja, em disponibilizar os dados de forma aberta. Para melhor explicação, o Quadro 4 aborda as cinco estrelas do Linked Open Data.

\section{Quadro 4 - Cinco estrelas do Linked Open Data}

\begin{tabular}{|c|l|}
\hline$\star$ & $\begin{array}{l}\text { Disponível na Web em qualquer formato, mas com uma licença } \\
\text { aberta, para ser Open Data; }\end{array}$ \\
\hline$\star \star$ & $\begin{array}{l}\text { Disponível como dados estruturados legíveis por máquina (por } \\
\text { exemplo, Excel em vez de digitalização de uma imagem de uma } \\
\text { tabela); }\end{array}$ \\
\hline$\star \star \star$ & $\begin{array}{l}\text { Disponível como dados estruturados legíveis por máquina em } \\
\text { formato não proprietário (por exemplo, CSV); }\end{array}$ \\
\hline$\star \star \star \star$ & $\begin{array}{l}\text { Além das três primeiras estrelas, utilizar padrões abertos da W3C, } \\
\text { como RDF e SPARQL para identificar as coisas, de modo que as } \\
\text { pessoas possam relacionar seu material; }\end{array}$ \\
\hline$\star \star \star \star$ & $\begin{array}{l}\text { Todos os demais e vincular seus dados para outros de pessoas } \\
\text { para fornecer contexto. }\end{array}$ \\
\hline
\end{tabular}

Fonte: (BERNERS-LEE, 2006, não paginado, tradução nossa).

Entre as tecnologias básicas para publicação de dados ligados estão: Uniform Resource Identifier (URI), linguagens de marcação como Extensible Markup Language (XML), uso do Resource Description Framework (RDF) e para recuperação e utilização do SPARQL Protocol and RDF Query Language.

URI é um identificador único e pode ser expresso um Uniform Resource Locators (URL) ou Uniform Resource Name (URN). O URL é caracterizado por trazer informações de onde o recurso pode ser obtido. O URN fornece a identificação de um recurso. No entanto, não oferece informações de onde o recurso pode ser obtido (RAMALHO, 2006). A linguagem XML possibilita especificar a forma dos dados no documento, além de permitir definições semânticas (ALMEIDA, 2002). Ressalta-se que, atualmente, há diversas linguagens para estruturar os dados, como J-SON, RDFa, Turtle, entre outras.

Por outro lado, o RDF é uma infraestrutura que permite a codificação, troca e reutilização de metadados estruturados. Ademais, possibilita a interoperabilidade de metadados por meio das convenções comuns de semântica, sintaxe e estrutura. Destaca-se que o RDF não estipula semântica 
para cada descrição, mas fornece a capacidade para definirem elementos de metadados, conforme necessário (FERREIRA; SANTOS, 2013, p. 15).

Sobre outras tecnologias, como SPARQL e Web Ontology Language (OWL), Santarem Segundo (2014, p. 3870) define o SPARQL como "[...] um conjunto de especificações que fornecem linguagens e protocolos para consultar e manipular o conteúdo publicado em RDF na Web.". Neste contexto, a Web Ontology Language (OWL) é fundamental para a estruturação dos dados por meio das ontologias. De acordo com Santarem Segundo e Coneglian (2016), a OWL é uma linguagem de marcação semântica para a definição, a instanciação, a publicação e a partilha de ontologias na World Wide Web.

Com o intuito de reunir orientações, padronizar e estruturar os dados ligados, em 2014 foram divulgadas as melhores práticas para publicação de dados ligados voltados para órgãos governamentais (WORLD WIDE WEB CONSORTIUM, 2014). Destaca-se que tais práticas podem ser aplicadas a qualquer outro contexto. Complementar à essas boas práticas, posteriormente, em 2017, foram liberadas as melhores práticas para publicação de dados na Web. De acordo com World Wide Web Consortium (2017), os benefícios em publicar os dados abertos são: reuso, compreensão, capacidade de conexão, facilidade de descoberta, confiança, facilidade de acesso, interoperabilidade e facilidade de processamento.

A aplicação dessas orientações e reestruturação dos catálogos de bibliotecas em ambientes digitais é uma tendência, conforme apontado por Marcondes (2012), Arakaki e Santos (2017), Arakaki, Simionato e Santos (2017), Serra e Santarem Segundo (2017), Simionato (2017), Silva, Serra e Santarem Segundo (2018), Gonçalez, Arakaki, Andrade e Simionato (2019), Jesus e Castro (2019), dentre outros.

Na próxima seção discutir-se-á o eixo relacional do modelo LRM e dos princípios Linked Data.

\section{IFLA LRM E LINKED DATA}

A partir do modelo IFLA LRM e dos princípios Linked Data e tecnologias semânticas, apresentadas nos tópicos anteriores, esta seção expõe algumas 
ponderações sobre as possibilidades de implementação do modelo LRM no Linked Open Data.

Com a nova percepção de fragmentação do registro bibliográfico já proposta pelo FRBR, os relacionamentos entre as entidades tornaram-se primordiais para os registros bibliográficos. O modelo IFLA LRM vem para consolidar essa estrutura e facilitar a criação de relacionamentos e, como visto, a unificação dos modelos FRBR, FRAD e FRSAD.

Além disso, com relação aos atributos, há a possibilidade de que sejam representados e implementados de forma real:

- um atributo pode ser representado como um mero literal (uma string, um número): é isso que o OWL (Web Ontology Language) considera como "propriedades de tipo de dados";

- um atributo pode ser representado como um Uniform Resource Identifier (URI) apontando para uma fonte externa (um documento referencial ou normativo de qualquer tipo, como um arquivo de autoridade ou uma lista de valores codificados); nesse caso, poderia ter sido modelado como um relacionamento, e não como um mero atributo, mas o modelo deve permanecer independente do modo como deve ser implementado: é isso que a OWL considera como "propriedades do objeto". Alguns atributos podem ser representados de qualquer maneira, outros podem ser representados apenas como literais; para aqueles que só podem ser representados como URls, a preferência era modelá-los como relacionamentos. (RIVA; BOEUF; ŽUMER, 2017a, p. 11).

Dessa forma, destaca-se que a viabilidade semântica de que os atributos será a partir da definição dos metadados que irão instanciar o modelo. Isto é, os metadados materializarão a estrutura proposta pelo modelo IFLA LRM. Ele deve ser usado como base para estruturação dos instrumentos de representação, como padrões e códigos de catalogação. "[...] Principalmente em termos de reutilização de dados em aplicações da Web semântica, tornando essa consideração parte integrante do planejamento inicial da apresentação da definição do modelo." (RIVA; BOEUF; ŽUMER, 2017a, p. 11).

Nesse sentido, o modelo IFLA LRM já foi instanciado para possíveis aplicações. O resultado dessa instanciação foi um modelo único, simplificado e logicamente consistente, que abrange todos os aspectos dos dados bibliográficos. Assim, da forma que o modelo LRM está sendo apresentado, ele 
se configura como um modelo conceitual. Porém, pela sua instanciação e a criação de namespaces, pode propiciar o mapeamento com outros padrões de metadados.

De acordo com Riva e Žumer (2016), algumas ações foram planejadas e como a criação de um namespace no domínio da IFLA LRM, esse domínio foi registrado no Open Metadata Registry (http://metadataregistry.org/) e estabelecido como Irmer (https://www.iflastandards.info//rm//rmer). O LRM Vocabularies também está disponível no GitHub pelo link https://github.com/iflastandards/LRM. O Quadro 5 mostra um trecho do registro a partir da instanciação da classe 'Obra' ('Work') do IFLA LRM.

\title{
Quadro 5 - Instância da Classe Obra do IFLA LRM
}

\begin{abstract}
$1<$--- Class: Work -->
$2<$ rdf:Description rdf:about="http://iflastandards.info/ns/lrm/lrmer/E2">

3 <rdfs:isDefinedBy rdf:resource="http://iflastandards.info/ns//rm/lrmer" />

4 <reg:status rdf:resource="http://metadataregistry.org/uri/RegStatus/1001" />

$5<$ rdfs:label xml:lang="en">Work</rdfs:label $>$

6 <reg:lexicalAlias rdf:resource="http://iflastandards.info/ns/lrm//rmer/Work" />

7 <skos:definition xml:lang="en">The intellectual or artistic content of a distinct creation</skos:definition>

$8<$ rdfs:comment xml:lang="en">A work is an abstract entity that permits the grouping of expressions that are considered functional equivalents or near equivalents. A work is a conceptual object, no single material object can be identified as the work. The essence of the work is the constellation of concepts and ideas that form the shared content of what we define to be expressions of the same work. A work is perceived through the identification of the commonality of content between and among various expressions. However, similarity of factual or thematic content alone is not enough to group several expressions as realizing the same instance of work. For example, two textbooks both presenting an introduction to calculus, or two oil paintings of the same view (even if painted by the same artist), would be considered distinct works if independent intellectual or artistic effort was involved in their creation. In the case of aggregating works and serial works, the essence of the work is the concept or plan for the selection, assembly and ordering of the expressions of other works to be embodied in the resulting aggregate manifestation. A work comes into existence simultaneously with the creation of its first expression, no work can exist without there being (or there having been at some point in the past) at least one expression of the work. A work can be recognized retrospectively from an examination of the individual realizations or expressions of the work. The work consists of the intellectual or artistic creation that lies behind all the various expressions of the work. As a result, the content identified with an instance of work can evolve as new expressions of it are created. Bibliographic and cultural conventions play a crucial role in determining the exact boundaries between similar instances of works. User needs are the basis for determining whether instances of expression are considered to belong to the same instance of work. When the majority of users, for most general purposes, would regard the expression instances as being intellectually equivalent, then these expressions are considered to be expressions of the same work. Generally, when a significant degree of independent intellectual or artistic effort is involved in the production of an expression, the result is viewed as a new work with a transformation relationship to the source work. Thus paraphrases, rewritings, adaptations for children, parodies, musical variations on a theme and free transcriptions of a musical composition are usually considered to represent new works. Similarly, adaptations of a work from one literary or art form to another (e.g., dramatizations, adaptations from one medium of the graphic arts to another, etc.) are considered to represent new works. Abstracts, digests and summaries are also considered to represent new works.</rdfs:comment>

9 <rdf:type rdf:resource="http://www.w3.org/2002/07/owl\#Class" />

$10<$ reg:name xml:lang="'>Work</reg:name>

11 <rdfs:subClassOf rdf:resource="http://iflastandards.info/ns//rm//rmer/E1" />

12 <owl:disjointWith rdf:resource="http://iflastandards.info/ns/lrm/lrmer/E3" />

13 <owl:disjointWith rdf:resource="http://iflastandards.info/ns//rm//rmer/E4" />

14 <owl:disjointWith rdf:resource="http://iflastandards.info/ns/lrm/lrmer/E5" />

15 <owl:disjointWith rdf:resource="http://iflastandards.info/ns//rm//rmer/E6" />

16 <owl:disjointWith rdf:resource="http://iflastandards.info/ns//rm//rmer/E9" />

17 <owl:disjointWith rdf:resource="http://iflastandards.info/ns//rm//rmer/E10" />

18 <owl:disjointWith rdf:resource="http://iflastandards.info/ns//rm/lrmer/E11" />
\end{abstract}

$19</$ rdf:Description $>$

Fonte: IFLA Namespaces (2006).

Conforme exposto no Quadro 5, logo na linha 1 é apresentado um comentário do que será descrito. $\mathrm{Na}$ linha 2, descreve-se o que se trata 0 recurso, ou seja, da entidade http://iflastandards.info/ns//rm//rmer/E2, que 
corresponde à classe 'obra', e utiliza a linguagem RDF. Na linha 3 é determinado pelo modelo apresentado, ou seja, do domínio Irmer (http://iflastandards.info/ns//rm/lrmer). Para descrever essa informação é utilizado o RDFs. Na linha 4 estabelece-se o status do recurso. Dessa forma, utiliza-se do namespace reg do Open Metadata Registry e rdf:resource que define o recurso. A linha 5 aponta o nome da classe e seu idioma, a partir do RDFS e XML.

Na linha 6 apresenta como o namespace reg e rdf:resource para definir http://iflastandards.info/ns//rm/lrmer/Work. Na linha 7 utiliza-se um namespace SKOS para esclarecer a definição da classe 'obra', e define pela estrutura do XML qual o idioma é apresentado o modelo. Na linha 8, há um comentário estruturado em RDFS, em língua inglesa, definido pelo xml:lang. Na linha 9, o tipo do objeto descrito em RDF, definindo como uma classe. Na linha 10 apresenta o nome work. A seguir, na linha 11, é utilizado o RDFS para dizer que a classe obra (work) é uma subclasse do RES. Por fim, nas linhas 12 a 18 é utilizado owl:disjoinWith para declarar que há uma disjunção das classes/entidades: expressão, manifestação, item, agente, nomem, lugar e intervalo de tempo.

O registro do namespace Irmer no Open Metadata Registry formaliza o IFLA LRM e permite que o modelo possa ser mapeado para outros metadados. De acordo com Riva e Žumer (2016, p. 6), o registro do namespace "[...] vai permitir ligações de codificação com os namespace FR existentes para permitir a interoperabilidade dos atuais sistemas bibliográficos com os novos, baseados em LRM.".

Diante deste cenário, é fundamental o estudo do mapeamento das classes do IFLA LRM com outros padrões, conforme apontado por Aalberg, Tallerås e Massey (2019). Assim, Taniguchi (2018) aponta algumas relações do modelo LRM utilizando a instanciação das classes e propriedades, conforme exposto na Figura 2. 


\section{Figura 2 - Visão do modelo LRM}

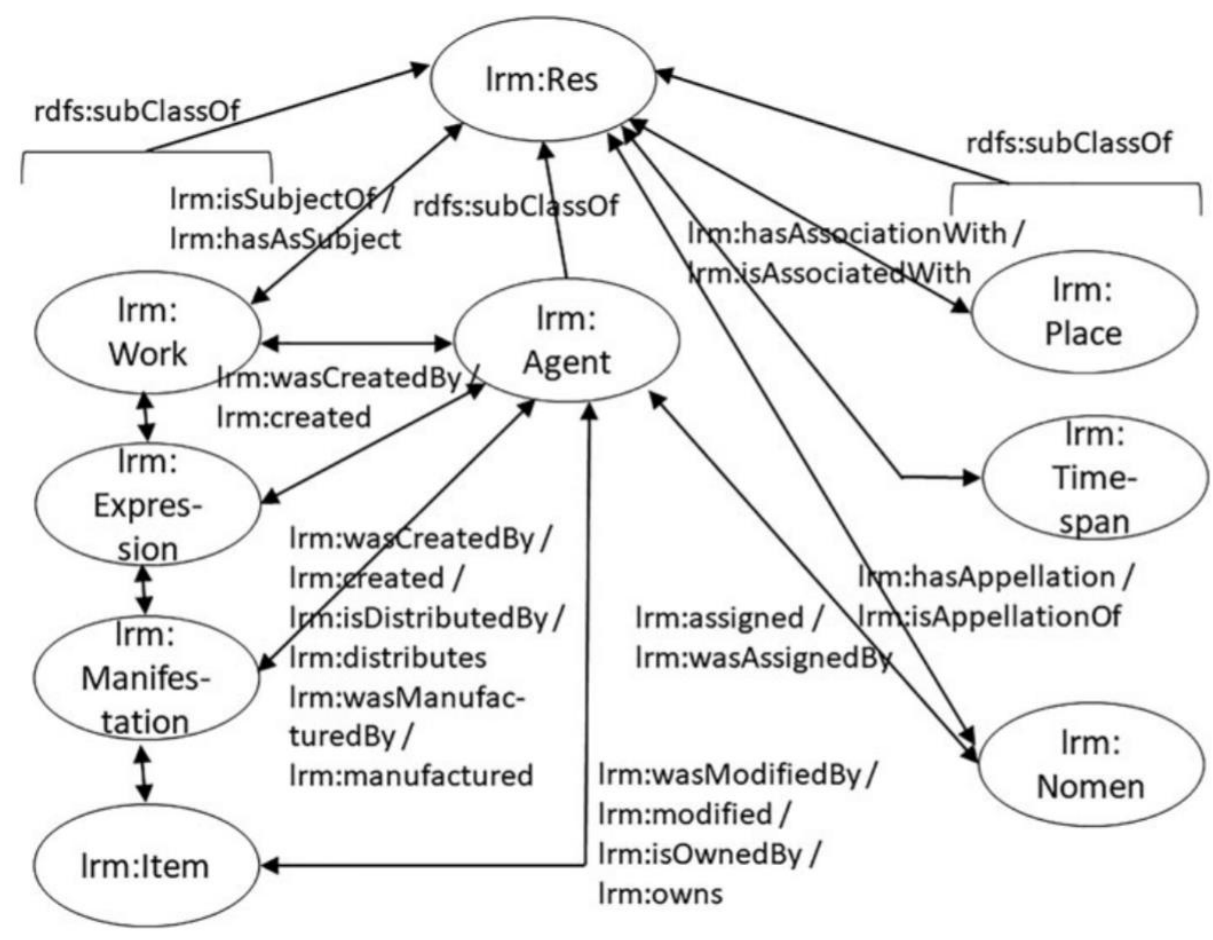

Fonte: Taniguchi (2018, p. 07)

A partir dos estudos de Taniguchi (2018, p. 27), o autor conclui que esses modelos e vocabulários possuem diferenças significativas, tais como: as formas de desenvolver as classes básicas para a representação de recursos, agentes e nomenclatura. Isto pode dificultar o mapeamento das classes, das entidades, das propriedades e dos atributos.

Nos estudos de Aalberg, Tallerås e Massey (2019, não paginado, tradução nossa)

Os resultados mostram que o modelo BIBFRAME tende a refletir e expressar a mesma estrutura de entidade que o modelo LRM, mas não fornece a mesma consistência e simplicidade na interligação e agrupamento de produtos intelectuais que podem ser alcançados com as entidades no modelo LRM.

Outros estudos abordaram a relação do IFLA LRM com a International Standard Bibliographic Description (ISBD) (INTERNATIONAL FEDERATION OF LIBRARY ASSOCIATIONS AND INSTITUTIONS, 2018) e com as diretrizes do Resource Description and Access (RDA) (RIVA, 2016; JONES, 2017; MERING, 2019; TEXEIRA; SHINTAKU; SCHIESSL; MURAKAMI; MACEDO, 2020).

O uso das boas práticas de publicação de dados na Web e do Linked Data 
pode ser aplicado a partir do modelo proposto. Essa documentação permite que desenvolvedores possam relacionar os conceitos propostos e aplicar em sistemas de informação. Os aspectos computacionais podem ser empregados a partir da definição e do uso dos metadados e dos relacionamentos, a partir das URIs.

\section{CONSIDERAÇÕES FINAIS}

Este artigo apresentou a harmonização dos modelos conceituais FRBR, FRAD e FRSAD, resultando no modelo IFLA LRM. O modelo IFLA LRM aparece como uma solução para inconsistências do FRBR, FRAD e FRSAD, a simplificar as entidades e tarefas dos usuários.

Expôs, ainda, brevemente algumas tecnologias semânticas, como URIs, linguagens de marcação, RDF, OWL, SPARQL, e os benefícios da publicação de dados em formato aberto. Conforme visto anteriormente, a implantação dessas tecnologias proporciona diversas vantagens no contexto do universo bibliográfico.

Pondera-se que, a instanciação do modelo LRM é um grande passo para a comunicação dos catálogos com a Web, e principalmente, com os usuários contemporâneos, que exigem uma resposta cada vez mais veloz. Em outro viés, essa iniciativa corrobora para um direcionamento do estudo e prática em Catalogação e Tecnologias semânticas, pois, agrega direcionamentos que antes pareciam inalcançáveis.

Apesar de a formalização e a instanciação do modelo serem recentes, já há alguns estudos que discutem sobre mapear as entidades apresentadas com outros padrões de metadados. Em suma, considera-se que, apesar do modelo ser recente, apresenta diversos estudos sobre o tema. Entretanto, as pesquisas sobre a relação entre o IFLA LRM e o Linked Data mostraram-se recentes e incipientes. Com eles, há diversos outros questionamentos que ainda precisam ser discutidos sobre a inserção de outras tecnologias semânticas em sua instanciação.

Acredita-se que, pela instanciação dos atributos do domínio Irmer, o conjunto de elementos das classes e propriedades RDF nativas descritas poderão ser significativas para a aplicação em catálogos digitais e que tenham o intuito dessa abertura dos registros e dados bibliográficos. 
Por fim, observa-se que os estudos futuros direcionam principalmente na aplicação dessa instanciação em registros de catálogos reais. Ainda, a evolução desses registros modelados e orientados pela estrutura LRM auxiliarão para a configuração dos catálogos em fontes de recuperação da informação mais modernas e mais rápidas.

\section{REFERÊNCIAS}

AALBERG, Trond; TALLERÅS, Kim; MASSEY, David. The impact of new bibliographic models on the search experience. In: INTERNATIONAL CONFERENCE ON CONCEPTIONS OF LIBRARY AND INFORMATION SCIENCE, 10., 2019, Ljubljana, Slovenia. Proceeding [...]. Ljubljana, Slovenia: Information Research, v. 4, n. 24, 2019. Disponível em: http://www.informationr.net/ir/24-4/colis/colis1915.html. Acesso em: 29 set. 2020.

\section{ALCTS. Core competencies for cataloging and metadata professional} librarians. Taiwan: LAROC, 2017. Disponível em:

http://www.lac.org.tw/files/core_competencies_cataloging_metadata_profession al.pdf. Acesso em: 29 set. 2020.

ALMEIDA, Maurício Bacellos. Uma introdução ao XML, sua utilização na Internet e alguns conceitos complementares. Ciência da Informação, Brasília, v. 31, n. 2, p. 5-13, 2002. Disponível em: http://dx.doi.org/10.1590/S010019652002000200001. Acesso em: 04 set. 2020.

ARAKAKI, Felipe Augusto. Linked Data: ligação de dados bibliográficos. 2016. Dissertação (Mestrado em Ciência da Informação - Faculdade de Filosofia e Ciências) - Universidade Estadual Paulista, Marília (SP), 2016. Disponível em: https://repositorio.unesp.br/handle/11449/147979. Acesso em: 04 set. 2020.

ARAKAKI, Felipe Augusto; SANTOS, Plácida Leopoldina Ventura Amorim da Costa. Linked Data em bibliotecas: iniciativas e tendências. In: ENCONTRO NACIONAL DE PESQUISA EM CIÊNCIA DA INFORMAÇÃO, 18., 2017, Marília. Anais [...]. Marília: UNESP; ANCIB, 2017. Disponível em: http://enancib.marilia.unesp.br/index.php/XVIII_ENANCIB/ENANCIB. Acesso em: 04 set. 2020.

ARAKAKI, Felipe Augusto; SIMIONATO, Ana Carolina; SANTOS, Plácida Leopoldina Ventura Amorim da Costa. Catalogação e tecnologia: interseções com a web semântica. Inf. Prof., Londrina, v. 6, n. 2, p. 03-19, 15 maio 2018. Disponível em: http://dx.doi.org/10.5433/2317-4390.2017v6n2p03. Acesso em: 04 set. 2020. 
BERNERS-LEE, T. Linked Data: Design Issues. [s.I.]: W3C, 2006. Disponível em: http://www.w3.org/Designlssues/LinkedData.html. Acesso em: 04 set. 2020.

COYLE, Karen. FRBR, before and after: a look at our bibliographic models. Chicago: American Library Association Editions, 2016.

FERREIRA, Jaider Andrade; SANTOS, Plácida Leopoldina Ventura Amorim da Costa. O modelo de dados Resource Description Framework (RDF) e o seu papel na descrição de recursos. Informação \& Sociedade: estudos, João Pessoa, v. 23, n. 2, p. 13- 23, 2013. Disponível em: https://periodicos.ufpb.br/ojs/index.php/ies/article/view/15436. Acesso em: 20 set. 2020.

\section{FUSCO, Elvis. Aplicação dos FRBR na modelagem de catálogos bibliográficos digitais. São Paulo: Cultura Acadêmica, 2011.}

GONÇALEZ, Paula Regina Ventura Amorim; ARAKAKI, Felipe Augusto; ANDRADE, Morgana Carneiro; SIMIONATO, Ana Carolina. Os benefícios do Linked Data para catálogos de bibliotecas: reflexões preliminares. In: CONGRESSO BRASILEIRO DE BIBLIOTECONOMIA E DOCUMENTAÇÃO, 28., 2019, Vitória/ES. Anais [...]. Viória/ES: FEBAB, 2019. Disponível em: https://portal.febab.org.br/anais/article/view/2049. Acesso em: 20 set. 2020.

INTERNATIONAL Federation of Library Associations and Institutions. Mapping from ISBD to IFLA LRM. Den Haag: IFLA, 2018. Disponível em:

https://www.ifla.org/files/assets/cataloguing/isbd/OtherDocumentation/mapping isbd-Irm_amd_2018_v.1.0.pdf. Acesso em: 20 set. 2020.

IFLA Namespaces. LRM Element Sets. 2020. Disponível em: https://www.iflastandards.info//rm/lrmer.html. Acesso em: 05 out. 2020.

IFLA Study Group on the Functional Requirements for Bibliographic Records. Functional Requirements for Bibliographic records: final report. [s.I.]: IFLA, 2009. Disponível em:

https://www.ifla.org/files/assets/cataloguing/frbr/frbr_2008.pdf. Acesso em: 20 set. 2020.

JESUS, Ananda Fernanda de; CASTRO, Fabiano Ferreira de. Dados bibliográficos para o linked data: uma revisão sistemática de literatura. Brazilian Journal of Information Science, [s.I.], v. 13, n. 1, p. 45-55, 2019. Disponível em: https://doi.org/10.36311/1981-1640.2019.v13n1.08.p45. Acesso em: 3 out. 2020 .

JONES, Edgar. The evolution of the serial work, the FRBR Conceptual Model, and RDA. Cataloging \& Classification Quarterly, [s.I.], v. 56, n. 2-3, p. 128145, 2017. Disponível em: https://doi.org/10.1080/01639374.2017.1388325. Acesso em: 20 set. 2020. 
MARCONDES, Carlos Henrique. "Linked data" - dados interligados - e interoperabilidade entre arquivos, bibliotecas e museus na web. Encontros Bibli: revista eletrônica de biblioteconomia e ciência da informação, [s.l.], v. 17, n. 34, p. 171-192, 2012. Disponível em: https://doi.org/10.5007/15182924.2012v17n34p171. Acesso em: 07 set. 2020.

MAXWELL, Robert L. FRBR: a guide for the perplexed. Chicago: American Library Association Editions, 2008.

MERING, Margaret. IFLA Library Reference Model, RDA, and serials in a nutshell. Serials Review, [s.l.], v. 45, n. 1-2, p. 66-68, 2019. Disponível em: https://doi.org/10.1080/00987913.2019.1627691. Acesso em: 7 set. 2020.

RAMALHO, Rogério Aparecido Sá. Web Semântica: aspectos interdisciplinares da gestão de recursos informacionais no âmbito da ciência da informação. 2006. 120 f. Dissertação (Mestrado - Faculdade de Filosofia e Ciências) - Universidade Estadual Paulista, Marília (SP), 2006. Disponível em: http://repositorio.unesp.br/handle/11449/93709. Acesso em: 7 set. 2020.

RIVA, Pat. Building RDA using the FRBR Library Reference Model. In: IFLA WLIC, 2016, Columbus/OH. Anais [...]. Columbus/OH: IFLA, 2016. Disponível em: http://library.ifla.org/1911/1/S17-2016-riva-en.pdf. Acesso em: 7 set. 2020.

RIVA, Pat; ŽUMER, Maja. The IFLA Library Reference Model, a step toward the Semantic Web. In: IFLA WLIC, 2016, Columbus/OH. Anais [...]. Columbus/OH: IFLA, 2016. Disponível em: http://library.ifla.org/1763/1/078-riva-en.pdf. Acesso em: 7 set. 2020.

RIVA, Pat; BœUF, Patrick Le; ŽUMER, Maja. IFLA Library Reference Model: um modelo conceitual para a informação bibliográfica. Tradução de Isabel Cristina Ayres da Silva Maringelli, José Fernando Modesto da Silva, Liliana Giusti Serra, Luiza Wainer, Marcelo Votto Texeira, Raildo de Sousa Machado e Zaira Regina Zafalon. Den Haag. IFLA, 2017a. Título original: IFLA Library Reference Model: a conceptual model for bibliographic information. Disponível em: https://www.ifla.org/files/assets/cataloguing/frbr-Irm/ifla-Irm-august2017_rev201712-por.pdf. Acesso em: 7 out. 2020.

RIVA, Pat; BœUF, Patrick Le; ŽUMER, Maja. IFLA LRM Transition Mappings. Den Haag: IFLA, 2017b. Disponível em:

https://www.ifla.org/files/assets/cataloguing/frbr-

Irm/transitionmappings201708.pdf. Acesso em: 01 out. 2020.

SANTAREM SEGUNDO, José Eduardo. Web semântica: introdução a recuperação de dados usando SPARQL. In: ENCONTRO NACIONAL DE PESQUISA EM CIÊNCIA DA INFORMAÇÃO, 15., 2014, Belo Horizonte/MG. Anais [...]. Belo Horizonte/MG, ANCIB; UFMG, 2014. Disponível em: http://enancib2014.eci.ufmg.br/documentos/anais/anais-gt8. Acesso em: 01 set. 2020. 
SANTAREM SEGUNDO, José Eduardo; CONEGLIAN, Caio Saraiva. Web Semântica e Ontologias: um estudo sobre construção de axiomas e uso de inferências. Informação \& Informação, Londrina, v. 21, n. 2, p. 217-244, 2016. Disponível em: http://dx.doi.org/10.5433/1981-8920.2016v21n2p217. Acesso em: 28 set. 2020.

SANTOS, Plácida Leopoldina Ventura Amorim da Costa. Catalogação e ética no ambiente colaborativo e de empoderamento das redes informacionais. In: FREIRE, Gustavo Henrique de Araujo (org.). Ética da informação: conceitos, abordagens, aplicações. João Pessoa: Ideia, 2010.

SERRA, Liliana Giusti; SANTAREM SEGUNDO, José Eduardo. O catálogo da biblioteca e o linked data. Em Questão, [s.I.], v. 23, n. 2, p. 167-185, 2017. Disponível em: https://doi.org/10.19132/1808-5245232.167-185. Acesso em: 28 set. 2020.

SILVA, Luciana Candida da; SERRA, Liliana Giusti; SANTAREM SEGUNDO, José Eduardo. O ORCID como aplicação de Linked Data no catálogo de bibliotecas. Informação \& Tecnologia, [s.I.], v. 4, n. 2, p. 185-205, 2018. Disponível em: https://doi.org/10.22478/ufpb.2358-3908.2017v4n2.40186. Acesso em: 29 set. 2020.

SIMIONATO, Ana Carolina. Família FR, BIBFRAME e Linked Data: integração de dados bibliográficos. In: ZAFALON, Zaira Regina; DAL'EVEDOVE, Paula Regina. (orgs.). Perspectivas da representação documental: discussões e experiências. São Carlos/SP: CPOI, 2017, v. 1, p. 211-226.

TANIGUCHI, Shoichi. Mapping and merging of IFLA Library Reference Model and BIBFRAME 2.0. Cataloging \& Classification Quarterly, [s.I.], v. 56, n. 56, 2018. Disponível em: https://doi.org/10.1080/01639374.2018.1501457. Acesso em: 29 set. 2020.

TEXEIRA, Marcelo Votto; SHINTAKU, Milton; SCHIESSL, Ingrid Torres; MURAKAMI, Tiago Rodrigo Marçal; MACEDO, Diego José. Análise prática do Recurso Descrição e Acesso e Modelo de Referência Bibliotecária no Koha no processo de catalogação. Informação \& Sociedade: estudos, João Pessoa, v. 30, n. 3, p. 1-14, 2020. Disponível em: https://doi.org/10.22478/ufpb.18094783.2020v30n3.54063. Acesso em: 01 out. 2020.

WORLD WIDE WEB CONSORTIUM. Best Practices for Publishing Linked Data. [s.I.]: W3C, 2014. Disponível em: https://www.w3.org/TR/ld-bp/. Acesso em: 01 out. 2020.

WORLD WIDE WEB CONSORTIUM. Boas Práticas para Dados na Web. São Paulo: W3C, 2017. Disponível em: https://www.w3.org/Translations/DWBP-ptBR/. Acesso em: 01 out. 2020.

ŽUMER, Maja. IFLA Library Reference Model (IFLA LRM): harmonisation of the frbr family. In: HJØRLAND, Birger; GNOLI, Claudio (comp.). Encyclopedia of 
Knowledge Organization. Kent/Ohio: ISKO, 2019. p. 1. Disponível em: https://www.isko.org/cyclo/lrm. Acesso em: 01 out. 2020.

\title{
IFLA LIBRARY REFERENCE MODEL AND LINKED DATA
}

\begin{abstract}
Introduction: In view of the new proposal of the IFLA LRM, the conceptual models of the FR family are consolidated to facilitate the creation of relationships between entities and in the definition of attributes. However, LRM's makes changes in its approach to the availability of data and the Semantic Web. Objective: Thus, the objective is to present the IFLA LRM and relate it to the new technologies available to enable the instance of the model from the concepts of the Semantic Web. Methodology: It is characterized by a qualitative and exploratory research, through a bibliographic survey on the IFLA LRM and linked data principles, with the clipping of research in international and national works since the year of publication of the LRM. Results: The main contribution is explained by the instantiation of the class 'work' and discussions are presented about the relationships of the IFLA LRM. Conclusions: It is considered that, by instantiating the attributes of the 'Irmer' domain, the studies may be significant for the application in digital catalogs and that they aim at this opening of records and bibliographic data.
\end{abstract}

Descriptors: IFLA Library Reference Model. Linked Data. Semantic Web. Conceptual models.

\section{MODELO DE REFERENCIA BIBLIOTECARIA DE LA IFLA Y LOS DATOS VINCULADOS}

\begin{abstract}
RESUMEN
Introducción: En vista de la nueva propuesta del modelo IFLA LRM, los modelos conceptuales de la familia FR se consolidan con el fin de facilitar la creación de relaciones entre entidades y en la definición de atributos. Además, el LRM trae cambios en su enfoque a la disponibilidad de los datos y la Web Semántica. Objetivo: El objetivo es presentar el modelo LRM de la IFLA y relacionarlo con las nuevas tecnologías disponibles para permitir la instanciación del modelo a partir de los conceptos de la Web Semántica. Metodología: Se caracteriza por una investigación cualitativa y exploratoria, a través de una encuesta bibliográfica sobre el modelo LRM de la IFLA y los principios de datos vinculados, con el recorte de la investigación en obras internacionales y nacionales desde el año de publicación del modelo LRM. Resultados: La contribución principal se explica por la creación de instancias del "obra" de la clase y se presentan discusiones sobre las relaciones del modelo LRM. Conclusiones: Se considera que, al crear instancias de los atributos del dominio 'Irmer', los estudios pueden ser significativos para la aplicación en catálogos digitales y que tienen como objetivo esta apertura de registros y datos bibliográficos.
\end{abstract}

Descriptores: Modelo de Referencia Bibliotecaria de la IFLA. Datos vinculados. Web Semántica. Modelos conceptuales. 
Recebido em: 15/09/2020

Aceito em: 30/09/2020 